\title{
Editorial
}

\section{Allergic Rhinitis and the Otolaryngologists}

Nearly all ENT specialists consider allergic Rhinitis (AR) as a common condition which is about $30 \%$ of their patients in private practices. The conventional method of treatment is practiced by all of them. They like to prescribe steroid spray locally, antiallergic, $\mathrm{H}_{2}$ receptors blocker, even calcium containing drugs.

The aim of this article is to prepare practitioners to face that patient with AR, not its treatment.

Diagnosis of AR is very easy, take care of AR or to advise the patient is not costly but to follow the advises of doctor fully is hardly possible for patients.

Not too long ago, much of the medical community and a large segment of the common people considered allergy a questionable condition at best. The opinion "It's all in their head" was often expressed when the diagnosis of allergy was suggested.

Foods that a patient dislikes are often represented as foods to which the patient is allergic. Unpleasant working conditions may be reported as places harboring substances to which the worker is allergic. Allergy is also blamed for poor academic performance in schools. Whereas formerly clinicians were often reluctant to make a diagnosis of nasal allergy, they may now frequently find it necessary to modify a patient's conviction that some form of allergy is at the root of all problems.

A conscientious physician, aware of the prevalence of allergy, must become at least reasonably knowledgeable as to the true extent of the problem and proper approaches to diagnosis and care.
To address properly the need for becoming involved in treating allergy, the physician must ask certain critical questions: How important is allergy for a practicing clinician? Is it truly widespread and debilitating enough to affect the practice?

Although generally not life-threatening, from an economic point of view allergy is not a minor problem. Based on figures reported in 1980, in USA allergic rhinitis alone had an expenditure of $\$ 224$ million annually in physicians' services, and an additional \$297 million for relief of symptoms. The same condition produced 2 million days of absence from schools annually, and 3.5 million people lost work days, accounting for \$ 154 million annually in lost wages.

Current estimates are that allergy in one form or another affects some $30 \%$ or more of the general population. Otolaryngologists may expect about $50 \%$ of the patients with symptoms of allergy as a major or at least a contributing factor of the illness.

To make the diagnosis, the clinician must be alert for allergy. The manifestations are multiple but frequently not obvious unless specifically sought. Allergy has been called "the great masquerader" because of its ability to mimic an immense variety of other conditions.

If the problem starts before the age of 1 year, in roughly $80 \%$ of cases the child will be found to be allergic. (In most cases, when this appears in infancy, the culprit is food. It can be distressing to find that simple dietary control might have saved repeated myringotomies with tubes, and the attendant risks.) The adult who complains of repeated 
respiratory infections every month or so, especially without fever, requires an allergic evaluation. Many cases of migraine-type headache are actually allergic in origin. A wide variety of gastrointestinal complaints may actually be food hypersensitivities. In short, almost any medical condition may be imitated by allergy.

It is not necessary to understand fully all the underlying mechanisms by which allergy affects the body. (This is fortunate, as science does not as yet fully understand all these mechanisms.) Nor is it necessary to keep at one's fingertips all the principles of each test devised for the diagnosis of allergy. What is necessary is a basic, general knowledge of the immunologic mechanisms governing allergy as far as they are understood at present, and of how these mechanisms apply to allergy testing and treatment. Details, as presented here, should be gone over and understood, but they may then be relegated to the realm of "know where to find when needed."

The ears, nose, and throat are the portals of entry for all allergens, and because four of the five senses are based predominantly in the ear, nose, and throat area, a major relationship would be expected and does in fact exist. Conditions that offend the senses drive the patient to the physician, often more rapidly than more dangerous conditions. This probably contributes to the frequency of ENT visits for conditions caused by allergy.
Symptoms are embarrassing, suppression of the symptoms temporarily is not difficult but reappearance is cumbersome. However days are not an away when immunotherapy and some newer methods of treatment will beat AR.

Despite the advances in allergy care during the past several decades, there are still only three basic accepted approaches to allergy care:

a. Avoidance (not to go in contact or geographic move)

b. Pharmacotherapy

C. Immunotherapy. Today's patient must opt for one or more of the established approaches presently available to reduce or alleviate sneezing, itching rhinorrhea or congestion. Pharmacotherapy with corticosteroid may give dramatic result for time being but must be aware of the side effects. Allergic rhinitis may precipitate asthma and at the same time literature reported tonsillectomy in case of allergic rhinitis may invite asthma. Minor surgery with the aim to change the nasal mucosal pattern may be practiced with close follow up.

\section{Prof. Dr. Pran Gopal Datta}

Professor of Otolaryngology- Head and Neck Surgery,

Former Vice Chancellor, BSMMU, Dhaka, Bangladesh 